\title{
Composition floristique et potentiel fourrager des principaux ligneux des parcours d'accueil des transhumants dans la Basse et Moyenne Vallée de l'Ouémé en zone guinéo-soudanienne du Bénin.
}

\author{
Sylvanus H. S. HONVOU1, André Boya ABOH'1,2, Oscar TEKA ${ }^{1}$, Christophe Bernard GANDONOU3, \\ Madjidou OUMOROU1,4, Guy Apollinaire MENSAH ${ }^{5}$, Brice SINSIN1 \\ 'Laboratoire d'Écologie Appliquée, Faculté des sciences Agronomiques/Université d'Abomey-Calavi 01 BP 526 \\ Cotonou, Bénin \\ ${ }^{2}$ College of livestock management and operating system, National University of Agriculture, Republic of Benin (West \\ Africa), BP 90 Kétou, Republic of Benin. \\ ${ }^{3}$ Laboratoire de Physiologie Végétale et d'Étude des Stress Environnementaux, Faculté des Sciences et Techniques, \\ Université d'Abomey-Calavi, 01BP 526 Tri postal, Cotonou, République du Bénin \\ ${ }^{4}$ Département de Génie de l'Environnement, École Polytechnique d'Abomey-Calavi/Université d'Abomey-Calavi, 01 \\ BP 5262009 Cotonou, Bénin. \\ 5Laboratoire de Recherché Zootechnique Vétérinaire et Halieutique /Institut National des Recherché Agricoles du \\ Bénin, 01 BP 884 Cotonou, Bénin. \\ *Corresponding author, E-mail : aboh.solex@gmail.com ; Tel. : (00 229) 97931422
}

Original submitted in on 21st June 2018. Published online at www.m.elewa.org on $30^{\text {th }}$ November 2018 https://dx.doi.org/10.4314/jab.v131i1.2

\section{RÉSUMÉ}

Objectifs : Les ligneux fourragers constituent une ressource alimentaire dans la zone d'accueille des bovins transhumants en saison sèche. L'objectif de cette étude était d'inventorier les ligneux fourragers des parcours des transhumants de la Basse et Moyenne Vallée de l'Ouémé, d'évaluer leur contribution pour l'alimentation des bovins transhumants en saison sèche et d'analyser leurs modes d'utilisation en vue de l'amélioration de la gestion de l'écosystème.

Méthodologie et Résultats : Des suivis des troupeaux au pâturage ont été effectués pour identifier les principaux ligneux fourragers. Les mesures sur les ligneux ont été réalisées dans 181 placeaux de $50 \mathrm{mX}$ $50 \mathrm{~m}$ installées au hasard selon le type de sol et de végétation. La méthode non destructive été a utilisée pour évaluer la biomasse foliaire. Pterocarpus santalinoides (Teck africain), Kigelia africana (Saucissonier), Lonchocharpus sericeus (Lilas du Sénégal) Mitragyna inermis (Pied d'éléphant), Daniellia oliveri (baumier d'llorin) et Vitex doniana (Prunier noir) étaient les ligneux présentant un grand intérêt pastoral. La biomasse foliaire a varié de 5 à $346,90 \mathrm{~kg} \mathrm{MS} /$ ha et la capacité de charge de 0,003 à 0,185 unité de bétail tropical par hectare. Le mode d'exploitation des ligneux est basé sur l'élagage des branches et l'abattage.

Conclusions et application des résultats : La présente étude a montré que les ligneux fourragers tels que $D$. oliveri, $K$. africana, L. sericeus, $M$. inermis, $P$. santalinoides et $V$. doniana sont prioritaires pour l'alimentation des bovins transhumants pendant la saison sèche. Cependant, les modes d'utilisation et le surpâturage compromettent leur régénération naturelle. Il est nécessaire d'améliorer la gestion durable des pâturages afin de continuer à alimenter le bétail pendant la saison sèche. II est également important de développer des moyens efficaces de régénération de ces espèces ligneuses en utilisant des politiques de gestion participative des ressources mises en œuvre pour assurer la durabilité des services sociaux et pastoraux. Mots clés: ligneux fourragers, caractéristique structurale, biomasse foliaire, capacité de charge, végétation, Bénin 


\section{ABSTRACT}

Floristic composition and forage potential of the main tree fodder of transhumant rangeland in the Lower and Medium Oueme Valley in the Guineo-Sudanean zone of Benin

Objectives: Tree forage is a feed resource in area receiving the transhumant ruminants, during the dry season. The objective of this study was to inventory fodder trees of the rangelands of the Lower and Mean Valley of Oueme, to evaluate their contribution to animal feeding and to analyze the modes of use in order for better management of the ecosystem.

Methodology and Results: Grazing surveys were conducted to identify the main forage trees. The data were collected in 181 plots of $50 \mathrm{~m}$ X $50 \mathrm{~m}$ installed randomly according to type of soil and vegetation. The nondestructive method was used to evaluate tree leaf biomass. Fodder tree plants such as Pterocarpus santalinoides (African teak), Kigelia africana (Sausage tree), Lonchocharpus sericeus(Senegal lilac) Mitragyna inermis(Elephant foot), Daniellia oliveri (West African copal) and Vitex doniana (Black plum tree) have shown great pastoral interest. Leaf biomass varied from 5 to $346.90 \mathrm{~kg}$ MS / ha and carrying capacity from 0.003 to 0,185 Tropical Livestock Unit per ha. The mode of use of the trees leaves is based on the pruning of the branches and the felling.

Conclusions and application of findings : The present study showed that the fodder trees such as $D$. oliveri, $K$. africana, L. sericeus, $M$. inermis, $P$. santalinoides, and $V$. doniana are priorities for rangelands feeding during dry season by transhumant farmer. However, the modes of use and the overgrazing of trees, compromise their natural regeneration. There is a need to improve sustainable management of rangelands in order to continue to feed the cattle during the dry season. So, it's also important to develop effective means of regeneration of these woody species by using participatory resource management policies implemented to ensure sustainability of social and pastoral services.

Keywords : Ligneous forage, structural characteristic, leaf biomass, carrying capacity, vegetation, Benin

\section{INTRODUCTION}

Au Bénin, comme dans la plupart des pays en Afrique au Sud du Sahara, l'élevage des ruminants domestiques pratiqué est de type pastoral. Les enjeux actuels pour les élevages d'herbivores sont d'arriver à concilier l'efficacité de la production avec des attentes sociétales fortes, notamment visà-vis de la qualité des produits, du bien-être animal et de la préservation de l'environnement (Ginane et al., 2008). Pour ce système d'élevage extensif, l'alimentation des animaux provient essentiellement de l'exploitation des parcours naturels par le biais de la conduite des troupeaux aux pâturages, et à la pratique de la transhumance (Kiema et al., 2014). La pratique de la transhumance a constitué pendant longtemps pour la majorité des éleveurs sahéliens, une stratégie efficace d'adaptation aux aléas climatiques et un système d'exploitation opportuniste des ressources pastorales face aux crises fourragères saisonnières, en tirant parti de la diversité écologique et de la complémentarité entre les différentes zones agro-climatiques du pays (Kiema et al., 2014). De plus en plus, cette transhumance est citée comme un moyen efficace d'adaptation aux changements climatiques en cours et de gestion durable des ressources naturelles (FAO, 2012). Malheureusement, avec la persistance de la variabilité climatique, la disponibilité et la productivité de la végétation spontanée varient dans le temps et dans l'espace. La production des herbacées n'arrivent plus à satisfaire les besoins nutritionnelles des animaux. La diminution de la biomasse fourragère et de la baisse progressive de la valeur nutritive des pâturages naturels en saison sèche entraînent des carences nutritionnelles chez les animaux (Breman et Ridder, 1991 ; Pamo et al., 2007). Les ligneux sont devenus la principale ressource fourragère pour le bétail en saison sèche à cause de leur teneur importante en protéine et de vitamines pour satisfaire les besoins d'entretien et de production des animaux (Njidda \& Ikhimioya, 2010 ; Mebirouk-Boudechiche et al., 2014 ; Sidi Imorou et al., 2016,). Les ligneux, les plus utilisés et étudiés comme fourrage par les éleveurs sont Khaya senegalensis (Cailceldrat), Afzelia africana (Luigué), Pterocarpus erinaceus (Palisandre du Sénégal), (Zampaligré et al., 2013 ; Sewadé et al., 2016). Les auteurs rapportent que ces ligneux connaissent aujourd'hui une importante régression sous l'effet combiné de la pression humaine et des 
perturbations climatique. La Basse et Moyenne Vallée de l'Ouémé (BMVO) située en zone guinéosoudanienne du Bénin, accueille pendant la saison sèche des troupeaux transhumants nationaux et transfrontaliers depuis plus de deux décennies (Alimi et al.,2015). Selon les auteurs, ce déplacement est motivé par l'abondance de l'eau et de fourrages dont surtout celui des ligneux. Malheureusement, les ligneux fourragers du parcours de cette zone d'accueil des transhumants a fait l'objet de peu d'étude en terme de caractéristique dendrométrique, d'importance pour

\section{MATÉRIELS ET MÉTHODES}

Présentation du milieu d'étude : La région de la Basse et Moyenne Vallée de l'Ouémé (BMVO) et sa Zone d'Influence (ZI) est localisée dans le Sud du Bénin entre $2^{\circ} 00$ et $2^{\circ} 45$ de longitude Est et $6^{\circ} 34$ et $7^{\circ} 32$ de latitude Nord (Figure1). Elle s'étend sur quatre départements du Bénin qui sont l'Ouémé, le Zou, l'Atlantique et le Plateau. Le climat est de type guinéen côtier équatorial (Adomou, 2005) dans la basse vallée, et celui de type guinéo soudanienne dans la partie moyenne de la vallée (Figure 1). Ce climat se caractérise par une longue saison pluvieuse de mars à juillet et une courte saison sèche en août ; une courte saison pluvieuse de septembre à novembre et une longue saison sèche de novembre à mars (Adam et Boko, 1993). La précipitation moyenne annuelle enregistrée de 1985 à 2012 était de $1130 \mathrm{~mm}$ à la station de Bohicon (Figure 2). Les températures moyennes annuelles a été de $28,1^{\circ} \mathrm{C}$ à Bohicon (ASECNA, 2016) (Figure 2). Le réseau hydrographique est constitué du fleuve Ouémé qui alimente le lac Nokoué à l'Ouest, la lagune de Porto-Novo à l'Est qui forme le delta de l'Ouémé avant de déboucher sur l'Océan Atlantique et son affluent Zou. La crue du fleuve s'amorce en juillet et atteint son niveau maximal en septembre et le retrait débute en octobre-novembre (Lalèyè et al., 2004). Les cultures de contre saison sont le pastoralisme, de production de biomasse foliaire et de modes d'exploitation. Face à ce gap d'information sur les ligneux fourragers dans les aires de terre de parcours des transhumants, la présente étude a pour objectif de documenter les ligneux fourragers de la BMVO en terme de composition floristique, de modes d'exploitation et de contribution des ligneux fourragers à l'alimentation des bovins et de l'analyse de l'interrelation entre la biomasse foliaire, les paramètres dendrométriques et la pédologie

très pratiquées par la population (Alimi et al., 2015, Atchadé et al., 2017). Selon Pelissier (1963), les coupes topographiques Est-Ouest de la vallée de l'Ouémé montrent deux unités géomorphologiques : i) Le plateau qui est une zone de formation latéritique très perméable. Il est parsemé de marécages dont certains ne s'assèchent pas au cours de l'année. ii) La basse plaine inondable qui est la zone de dépôt des alluvions apportées chaque année par les eaux de crue du fleuve Ouémé. Le milieu est caractérisé par les sols ferralitiques localisés sur les plateaux; les tourbes argileuses et tourbes brutes localisées au pied du plateau, les sols de bourrelet de berge argileux à limono-argileux et les sols humiques à gley (Paradis, 1983). La végétation est dominée par des mosaïques de cultures et jachères, de cultures et jachères sous palmier et de plantations forestières et fruitières. Cette mosaïque de cultures et jachères est combinée avec les savanes arborées, des savanes arbustives et savanes herbeuses. Les reliquats de forêts denses et de galeries forestières subsistent sous forme de petits ilots de forêts sacrées. Ce couvert végétal est le résultat d'une forte dégradation sous l'influence des actions anthropiques à travers l'exploitation agricole et les feux de brousse (Alimi et al., 2015). 


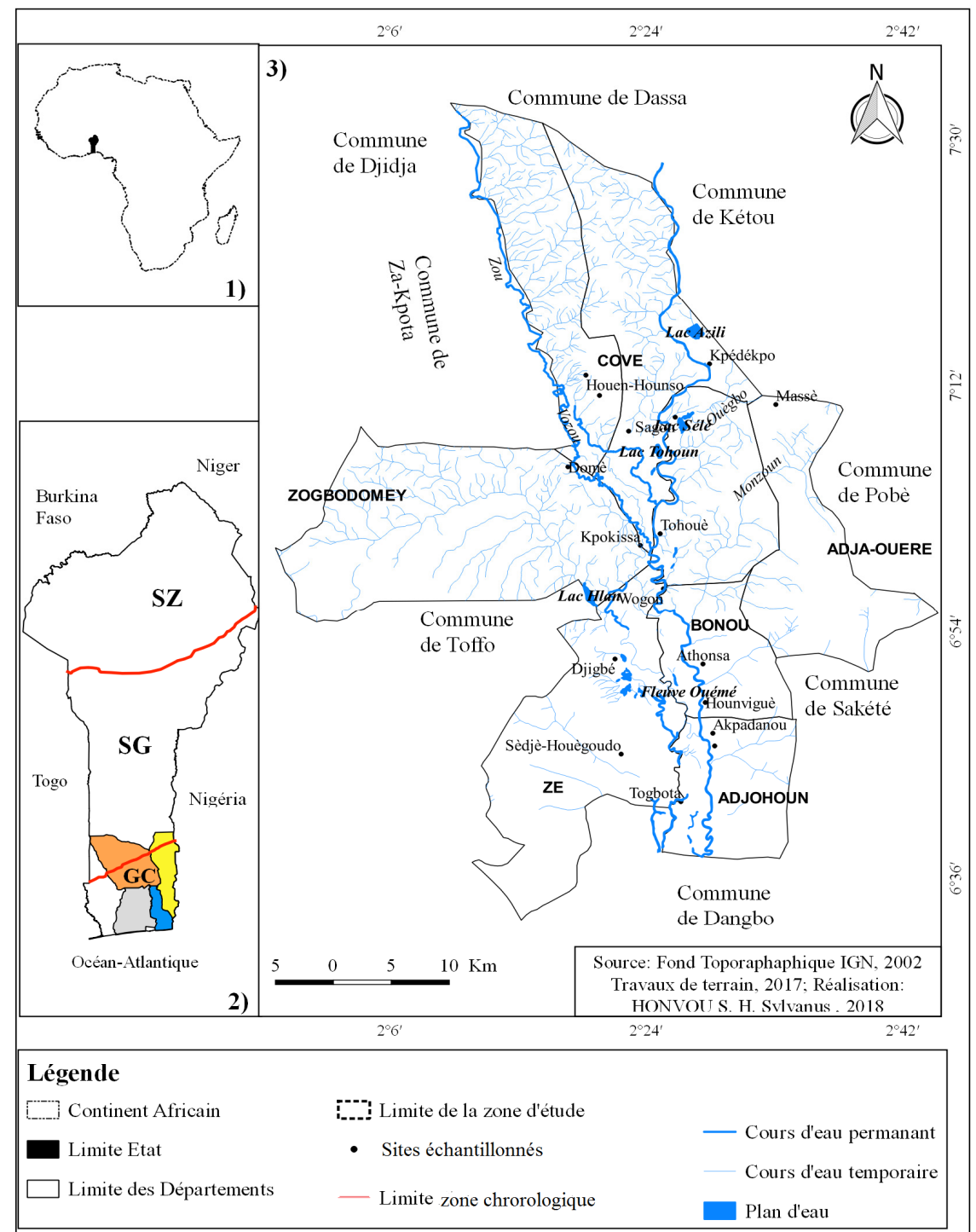

Figure 1 : Carte du Bénin montrant la zone d'étude et les différents sites échantillonnés

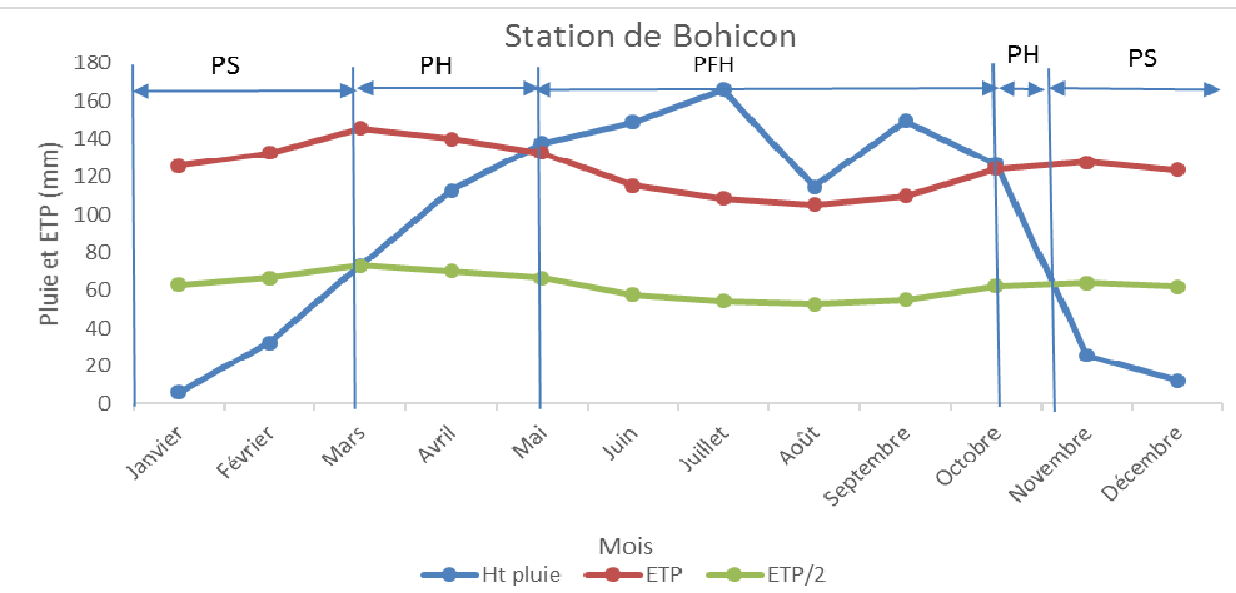

Figure 2 : Diagramme climatique de la zone d'accueil des transhumants de la BMVO à base des données de la station météorologique de Bohicon de 1985 à 2012.

$P S$ : Période sèche $(P<E T P / 2)$

$\mathrm{PH}$ : Période humide $(\mathrm{ETP} / 2<\mathrm{P})$

PFH : Période Franchement Humide $(E T P<P)$. 
Choix des sites et installation des placeaux : Les placeaux ont été installés dans 38 sites de la BMVO, qui ont accueilli des éleveurs transhumants pendant au moins 8 années au cours de la période allant de 2007 à 2017. Une prospection de la BMVO à l'aide de la carte d'occupation issue de l'image LANDSTAT ETM 2014 a été réalisée pour identifier les différents types de formations végétales. Cinq types de formations végétales que sont les champs, les jachères et la savane arborée, la savane arbustive et la savane herbeuse ont été inventoriés. Au total, 181 placeaux de $50 \mathrm{~m} \times 50 \mathrm{~m}$, ont été installés de façon aléatoire en tenant compte des types de formation végétale, de la topographie et du type de sol.

$$
F C=\frac{R i}{R} X 100
$$

Où $\mathrm{Ri}=$ nombre de relevée dans lequel l'espèce est présente et $R$ le nombre total de relevé $(R)$. Les ligneuses fourragères ont été identifiées au cours des suivis des bovins aux pâturages pour leur accorder des indices de qualité spécifique (Is) en tenant compte de l'ingestion volontaire par les bovins aux pâturages. Cet Indice de qualité a été choisi sur l'échelle de 0 à 4 (Sinsin, 1993) (4 : fourrage de bonne qualité ; 2 ou 3 : fourrage de qualité moyenne ; 1 : fourrage de qualité médiocre $; 0$ : refus de fourrage). Cette notion intègre l'acceptabilité car certaines espèces sont plus ou moins recherchées (Sinsin, 1993); la notion d'ingestibilité

\section{$D($ individu $/$ ha $)=\frac{N}{S}$}

Avec $\mathrm{N}$, le nombre d'individu dans le placeau et $\mathrm{S}$, la surface échantillonnée $(0,25 \mathrm{ha})$. La biomasse foliaire des ligneux fourragers prioritaires a été aussi évaluée dans cinq (5) placeaux par type de formation végétale, installés de façon aléatoire. La circonférence à $1,30 \mathrm{~m}$

$$
D_{1,30}=\frac{\text { Circonference }}{\pi}
$$

Ensuite, deux individus de chaque ligneux fourrager ont été choisis au hasard pour évaluer la biomasse foliaire. Le nombre total de branches par individu a été dénombré et la biomasse foliaire deux branches choisies au hasard ont été coupées et pesées à l'aide d'un peson de portée $5 \mathrm{~kg}$. Les classes de diamètres
Inventaire floristique et identification des ligneux fourragers prioritaires: Dans le but d'avoir une connaissance sur le peuplement ligneux de la BMVO, un inventaire de toutes les espèces ligneuses a été réalisé dans les placeaux de $50 \mathrm{~m} \times 50 \mathrm{~m}$ afin d'établir la liste floristique. Un herbier a été réalisé pour leur identification à l'Herbier National, au Jardin Botanique et au Laboratoire d'Écologie Appliquée de l'Université d'Abomey-Calavi. Cet inventaire a permis d'établir la richesse spécifique et la fréquence centésimale des espèces. Cette richesse spécifique (S) est le nombre total d'espèce présente dans la zone d'étude. La fréquence centésimale a été obtenue suivant la formule (1).

(Loiseau, 1988), elle-même liée à la digestibilité et à la valeur fourragère. Au total, 5 animaux de 10 troupeaux bovins ont été suivis. Les ligneux fourragers sont qualifiés de prioritaires lorsque leurs indices de qualité varie entre 2-4 et recherchés par les éleveurs.

Évaluation de la biomasse foliaire et capacité de charge des ligneux fourragers prioritaires: Les ligneux fourragers dont la circonférence à $1,30 \mathrm{~m}$ audessus du sol est supérieur à $32 \mathrm{~cm}$ ont été recensés afin de déterminer leur densité. La densité a été déterminée à partir de la formule suivante :

au-dessus du sol et la hauteur totale de ces ligneux fourragers ont été mesurées. Le diamètre à $1,30 \mathrm{~m}$ de hauteur a été déduit de la mesure de la circonférence à $1,30 \mathrm{~m}$ en utilisant la formule (3)

\section{(3)}

des branches ont variés de 2 à $12 \mathrm{~cm}$ conformément aux branches coupés par les transhumants. Des échantillons de $150 \mathrm{~g}$ de feuilles ont été prélevés par branche et mis à l'étuve à $70^{\circ} \mathrm{C}$ pendant 72 heures pour la détermination de matière sèche suivant la formule :

$$
\text { MS } \%=\frac{\text { Poids sec de l'échantilion }}{\text { Poids frais de l'échantizlon }} \times 100
$$

La biomasse foliaire sèche de chaque individu (BFInd) de ligneux a été calculée suivant la formule :

$$
B F I n d(K g M S / \text { pied })=B M b r \times N B r \times \% M S
$$


BFInd : Biomasse foliaire par individu ; BMbr : biomasse moyenne des branches coupées et $\mathrm{NBr}$ : Nombre de branche total comptées. MS : Matière sèche

\section{$B F T(K q M S / h a)=B F I n d \times D$.}

BFInd : Biomasse foliaire par individu et $\mathrm{D}$ : la densité des ligneux
L'estimation de la biomasse foliaire (BFT) sèche moyenne à l'hectare a été calculée grâce à la densité des espèces de ligneux fourragers suivant la formule :

Enfin, la capacité de charge (UBT/ha) de la saison sèche (120 jours) été calculée pour chaque espèce à l'aide de la formule.

$$
\mathrm{CC}(U B T / \mathrm{ha})=\frac{\text { Biomasse foliaire totale }(\mathrm{Kg} \mathrm{MS} / \mathrm{ha})}{2 \times 120 \text { jours } \times 6_{, 25} 25 \mathrm{MS} / \mathrm{UBT} / \mathrm{j}}
$$

Analyse statistique: Le logiciel R 3.3.2 (R Development Core Team, 2016) a été utilisé pour les analyses statistiques. Le modèle linéaire à effets fixes croisés, à 2 critères de classifications que sont le type de formation végétal (TFV) et des espèces ligneuses (Esp) a été utilisé pour évaluer les caractéristiques structurales et la biomasse foliaire des ligneux fourragers. Préalablement, la normalité des données est vérifiée grâce au test de Shapiro-Wilk., l'homogénéité des variances des facteurs par le test Levene. En cas de non significativité au seuil de 5\%

\section{RÉSULTATS}

Richesse et composition floristiques : L'inventaire floristique dans la BMVO a permis de recenser 41 espèces ligneuses repartis en 21 familles et 38 genres (Tableau 1). Les Leguminosae $(26,83 \%)$ sont les plus représentés, suivies des Rubiaceae $(12,20 \%)$ et des Euphorbiaceae $(7,32 \%)$ (Figure 3). L'analyse globale a révélé que Mitragyna inermis a enregistré la fréquence pour l'interaction entre les facteurs TFV et Esp, l'analyse est reprise sans tenir compte de l'effet de l'interaction. Lorsque les facteurs pris isolément sont significatifs, ils font l'objet de structuration moyenne grâce au test de Student, Newman et Keuls. Les facteurs espèce de ligneux, le type de formation végétale, le type de sol ont été combinés pour apprécier la variabilité dendrométriques et de la biomasse foliaire des ligneux suivant l'Analyse en Composantes Principales (ACP) avec la fonction "PCA" du package "FactoMineR" (Le et al., 2008).

la plus élevée (Tableau 1), suivi de Elaeis guineensis et Lonchocarpus sericeus. Les espèces Pterocarpus santalinoides et Daniellia oliveri ont enregistré une fréquence moyenne (10,5 à 17\%). Par contre Kigelia africana, Vitex doniana et Dialium guineense, sont faiblement représentés $(0,55$ à $3,3 \%)$.

Tableau 1 : Fréquence centésimale des différentes espèces ligneuses

\begin{tabular}{l|l|c}
\hline Familles & Espèces & Fréquence (\%) \\
\hline Anarcardinaceae & Anacardium occidentale L. & 1,10 \\
\hline Anarcardinaceae & Mangifera indica L. & 2,21 \\
\hline Annonaceae & Annona senegalensis Pers. & 1,10 \\
\hline Arecaceae & Elaeis guineensis Jacq. & 34,81 \\
\hline Asparagaceae & Asparagus africanus Lam. & 0,55 \\
\hline Bignoniaceae & Kigelia africana (Lam.)Benth & 3,31 \\
\hline Bignoniaceae & Newbouldia leavis (P. Beauv.) Seeman Ex Bureau & 3,31 \\
\hline Bombacaceae & Adansonia digita L. & 2,76 \\
\hline Bombacaceae & Ceiba pentadra (L.) Gaertn & 4,42 \\
\hline Leguminosae & Daniellia oliveri (Rolfe) Hucth. \& Dalziel & 10,50 \\
\hline Leguminosae & Dialium guineense Wild. & 0,55 \\
\hline Leguminosae & Senna siamea & 1,66 \\
\hline Combretaceae & Anogeissus leicarpus (DC.) Guill.\&Perr. & 7,73 \\
\hline Combretaceae & Combretum indicum (L.) De Filipps & 0,55 \\
\hline Ebenaceae & Diospyros mespiliformis Hochst. Ex A.DC. & 1,66 \\
\hline Euphorbiaceae & Bridelia ferruginea Benth. & 0,55 \\
\hline Euphorbiaceae & Antidesma venosum E. Mey. Ex Tul. & 6,08 \\
\hline Euphorbiaceae & Flueggea virosa (Roxb. ex Willd.) Voigt & 3,87 \\
\hline Leguminosae & Acacia auriculiformis A. Cunn. ex Benth. & 3,87 \\
\hline
\end{tabular}


Honvou et al., J. Appl. Biosci. 2018 Composition floristique et potentiel fourrager des principaux ligneux des parcours d'accueil des transhumants dans la Vallée de l'Ouémé en zone guinéo-soudanienne du Bénin.

\begin{tabular}{l|l|c}
\hline Leguminosae & Lonchocarpus sericeus (Poir.) Kunth & 31,49 \\
\hline Leguminosae & Pterocarpus erinaceus Poir. & 11,05 \\
\hline Leguminosae & Pterocarpus santalinoides L'Hérit. Ex DC & 17,13 \\
\hline Leguminosae & Millettia thoningii (Schumach. \&Thonn) Baker & 1,66 \\
\hline Loganiaceae & Anthocleista vogelii Planch. & 1,66 \\
\hline Malvaceae & Triplochiton scleroxylon K.Schum. & 0,55 \\
\hline Meliaceae & Azadirachta indica A. Juss. & 7,73 \\
\hline Leguminosae & Acacia sieberiana DC. & 6,63 \\
\hline Leguminosae & Albizia zyga & 2,21 \\
\hline Leguminosae & Parkia biglobosa (Jacq.) R.Br ex Benth & 3,31 \\
\hline Moraceae & Ficus sp & 1,10 \\
\hline Poaceae & Bambusa vulgaris Schrad. ex J.C. Wendl. & 0,55 \\
\hline Rubiaceae & Gardenia erubescens Stapf.\&Huctch & 1,66 \\
\hline Rubiaceae & Mitragyna inermis (Wild.) Kuntze & 51,93 \\
\hline Rubiaceae & Morinda lucida Benth. & 14,92 \\
\hline Rubiaceae & Pavetta corymbosa (DC.) F.N. Williams & 1,10 \\
\hline Rubiaceae & Sarcocephalus latifolius (Sm.) E.A.Bruce & 2,76 \\
\hline Sapindaceae & Lecaniodiscus cupanioides Planch. Ex Benth & 1,10 \\
\hline Sterculaceae & Cola gigantea A. Chev. & 2,21 \\
\hline Sterculaceae & Cola laurifolia Mast. & 0,55 \\
\hline Verbenaceae & Tectona grandis L. f. & 6,63 \\
\hline Verbenaceae & Vitex doniana Sweet & 2,76 \\
\hline
\end{tabular}

Caractéristiques structurales et modes d'exploitation des ligneux fourragers prioritaires: Les ligneux fourragers prioritaires (06) identifiés étaient Pterocarpus santalinoides, Kigelia africana avec 4 et 3 comme indice de qualité spécifique, et Lonchocharpus sericeus, Mitragyna inermis, Daniellia oliveri et Vitex doniana qui ont chacun 2 pour l'indice de qualité spécifique. Leur densité a varié de 4 à 47 individus /ha (Tableau 2). Mitragyna inermis a présenté la densité la plus élevée $(P<0,05)$, suivi de Pterocarpus santalinoides avec $23,20 \pm 4,28$ individus/ha. La plus faible densité a été enregistrée au niveau de $K$. africana et $V$. doniana. Le nombre moyen de branches a varié de 12 à 19. Ce nombre est similaire $(P>0,05)$ pour les différentes espèces. Les hauteurs ainsi que les diamètres ont été similaires $(P>0,05)$ pour tous les ligneux. Les modes d'exploitation des ligneux fourragers ont varié suivant les espèces. L'émondage des branches de faible diamètre $(2-12 \mathrm{~cm})$ est pratiqué au niveau de Pterocarpus santalinoides, Kigelia africana pour alimenter les bovins. Par contre, ce sont les jeunes feuilles issues de la régénération naturelle des ligneux comme Mitragyna inermis, Daniellia oliveri, Lonchocarpus sericeus et Vitex doniana qui ont été broutés par les animaux transhumants. En général, ces ligneux étaient abattus ou coupés par la population locale et rarement par les transhumants.

Tableau 2: Caractéristiques structurales (moyenne \pm erreur type) des ligneux fourragers

\begin{tabular}{l|c|c|c|c|c}
\hline $\begin{array}{l}\text { Ligneux } \\
\text { Fourragers }\end{array}$ & Is & $\begin{array}{c}\text { Densité } \\
\text { (pied/ha) }\end{array}$ & $\begin{array}{c}\text { Nombre } \\
\text { de branche }\end{array}$ & $\begin{array}{c}\text { Diamètre } \\
\text { tronc } \mathbf{( c m})\end{array}$ & $\begin{array}{c}\text { Hauteur } \\
(\mathbf{m})\end{array}$ \\
\hline Daniellia oliveri & 2 & $9,33 \pm 2 ; 15 \mathrm{~b}$ & $19,00 \pm 3,40$ & $19,84 \pm 1,46$ & $7,17 \pm 0,54$ \\
\hline Kigelia africana & 3 & $4,00 \pm 0,00 \mathrm{~b}$ & $12,50 \pm 2,79$ & $36,44 \pm 3,39$ & $8,20 \pm 1,99$ \\
\hline Lonchocarpus sericeus & 2 & $9,64 \pm 2,55 \mathrm{~b}$ & $12,12 \pm 3,03$ & $14,47 \pm 2,90$ & $7,45 \pm 1,40$ \\
\hline Mitragyna inermis & 2 & $47,39 \pm 7,63 \mathrm{a}$ & $13,52 \pm 2,85$ & $22,21 \pm 3,53$ & $6,86 \pm 1,59$ \\
\hline Pterocarpus santalinoides & 4 & $23,20 \pm 4,28 \mathrm{ab}$ & $12,10 \pm 2,69$ & $23,24 \pm 3,62$ & $6,86 \pm 0,84$ \\
\hline Vitex doniana & 2 & $4,00 \pm 0,00 \mathrm{~b}$ & $12,67 \pm 2,42$ & $37,13 \pm 2,88$ & $8,67 \pm 1,42$ \\
\hline $\mathrm{P}$ & & 0,04 & 0,93 & 0,07 & 0,69 \\
\hline
\end{tabular}

Is : Indice de qualité

$a, b$ : indique que les valeurs suivies des lettres différentes $(a, b)$ dans la même colonne sont significativement différentes. 
Biomasse foliaire et capacité de charge des ligneux : Les rendements en biomasse foliaire ont variés de 4 à $346 \mathrm{kgMS} / \mathrm{ha}$ (tableau 3). Ce rendement bien que similaire pour les ligneux $(P>0,05)$, est relativement élevé pour $M$. inermis. . La capacité de charge est similaire $(P>0,05)$ pour les ligneux fourragers, mais relativement élevé pour $M$. inermis. Du point de vue type de formation végétale, la biomasse foliaire moyenne totale a varié de 24 à $638 \mathrm{Kg} \mathrm{MS} / \mathrm{ha}$ (tableau 4). Elle est relativement plus élevée dans la savane arborée. La capacité de charge a varié de 0,01 à $0,34 \mathrm{UBT} / \mathrm{ha}$, mais plus élevée dans la savane arborée $(P<0,05)$.

Tableau 3 : Biomasse foliaire et capacité de charge des ligneux fourragers

\begin{tabular}{l|c|c|c}
\hline $\begin{array}{l}\text { Ligneux } \\
\text { fourragers }\end{array}$ & $\begin{array}{c}\text { Biomasse (Kg } \\
\text { MS/individu) }\end{array}$ & $\begin{array}{c}\text { Rendement (Kg } \\
\text { MS/ha) }\end{array}$ & $\begin{array}{c}\text { Capacité de charge } \\
\text { (UBT/ha) }\end{array}$ \\
\hline D. oliveri & $5,43 \pm 1,85$ & $57,36 \pm 7,13$ & $0,031 \pm 0,02$ \\
\hline Kigelia africana & $1,21 \pm 0,82$ & $4,85 \pm 1,65$ & $0,003 \pm 0,00$ \\
\hline Lonchocarpus sericeus & $5,14 \pm 2,93$ & $61,16 \pm 12,37$ & $0,033 \pm 0,02$ \\
\hline Mitragyna inermis & $4,94 \pm 2,13$ & $346,90 \pm 27,26$ & $0,185 \pm 0,07$ \\
\hline Pterocarpus santalinoides & $2,42 \pm 1,40$ & $62,04 \pm 8,02$ & $0,033 \pm 0,01$ \\
\hline Vitex doniana & $2,42 \pm 1,34$ & $15,38 \pm 2,68$ & $0,008 \pm 0,00$ \\
\hline P & 0,40 & 0,53 & 0,53 \\
\hline
\end{tabular}

UBT : Unité de Bétail Tropical ; \pm erreur type

Tableau 4: Variation de la biomasse foliaire et de la capacité de charge en fonction du type de formation végétale

\begin{tabular}{l|c|c|c}
\hline $\begin{array}{l}\text { Type de formation } \\
\text { végétale }\end{array}$ & $\begin{array}{c}\text { Biomasse (Kg } \\
\text { MS/individu) }\end{array}$ & Rendement (Kg MS/ha) & $\begin{array}{c}\text { Capacité de charge } \\
\text { (UBT/ha) }\end{array}$ \\
\hline Champ de culture & $8,16 \pm 2,80$ & $68,79 \pm 7,05 \mathrm{ab}$ & $0,04 \pm 0,01 \mathrm{ab}$ \\
\hline Jachère & $3,06 \pm 1,28$ & $24,09 \pm 4,62 \mathrm{~b}$ & $0,01 \pm 0,00 \mathrm{~b}$ \\
\hline Savane arborée & $6,48 \pm 2,68$ & $638,78 \pm 31,55 \mathrm{a}$ & $0,34 \pm 0,13 \mathrm{a}$ \\
\hline Savane arbustive & $2,36 \pm 1,79$ & $68,57 \pm 12,04 \mathrm{~b}$ & $0,04 \pm 0,02 \mathrm{~b}$ \\
\hline Savane herbeuse & $3,69 \pm 1,98$ & $65,10 \pm 10,65 \mathrm{~b}$ & $0,03 \pm 0,02 \mathrm{~b}$ \\
\hline $\mathrm{P}$ & 0,03 & 0,01 & 0,01 \\
\hline
\end{tabular}

$a, b$ : indique que les valeurs suivies des lettres différentes $(a, b)$ dans la même colonne sont significativement différentes.

Inter-relation entre la biomasse foliaire, les paramètres dendrométriques, les formations végétales et la pédologie : La synthèse de l'Analyse des Correspondances Principales (ACP) a montré que l'axe 1 explique les $65 \%$ des relations entre la biomasse, les caractéristiques dendrométriques des ligneux, la formation végétale et pédologique. Les deux premiers axes expliquent $85,21 \%$ de ces relations (Tableau 5). La figure 3 indique deux groupes distincts correspondants chacun au niveau de production de biomasse qui s'oppose. La biomasse foliaire des ligneux est significativement corrélée $(P<0,05)$ avec les caractéristiques dendrométriques, le type de sol et la formation végétale. La biomasse foliaire est élevée pour les ligneux ayant un important effectif de branches, une hauteur et un diamètre élevés. Par contre la biomasse est faible pour les ligneux ayant ces paramètres dendrométriques faibles. Ainsi, les ligneux tels que $L$. sericeus, $K$. africana, $M$. inermis, et $D$. oliveri ayant ces paramètres dendrométriques élevés notamment sur les sols argileux ou argilo-limoneux ont enregistré une production de biomasse foliaire plus élevée.

Tableau 5 : Pourcentage cumulé de variance expliquée

\begin{tabular}{l|l|l|l}
\hline Axes & 1 & 2 & 3 \\
\hline Variable (\%) & 65,33 & 19,88 & 8,21 \\
\hline Variables cumulés (\%) & 65,33 & 85,21 & 93,43 \\
\hline
\end{tabular}




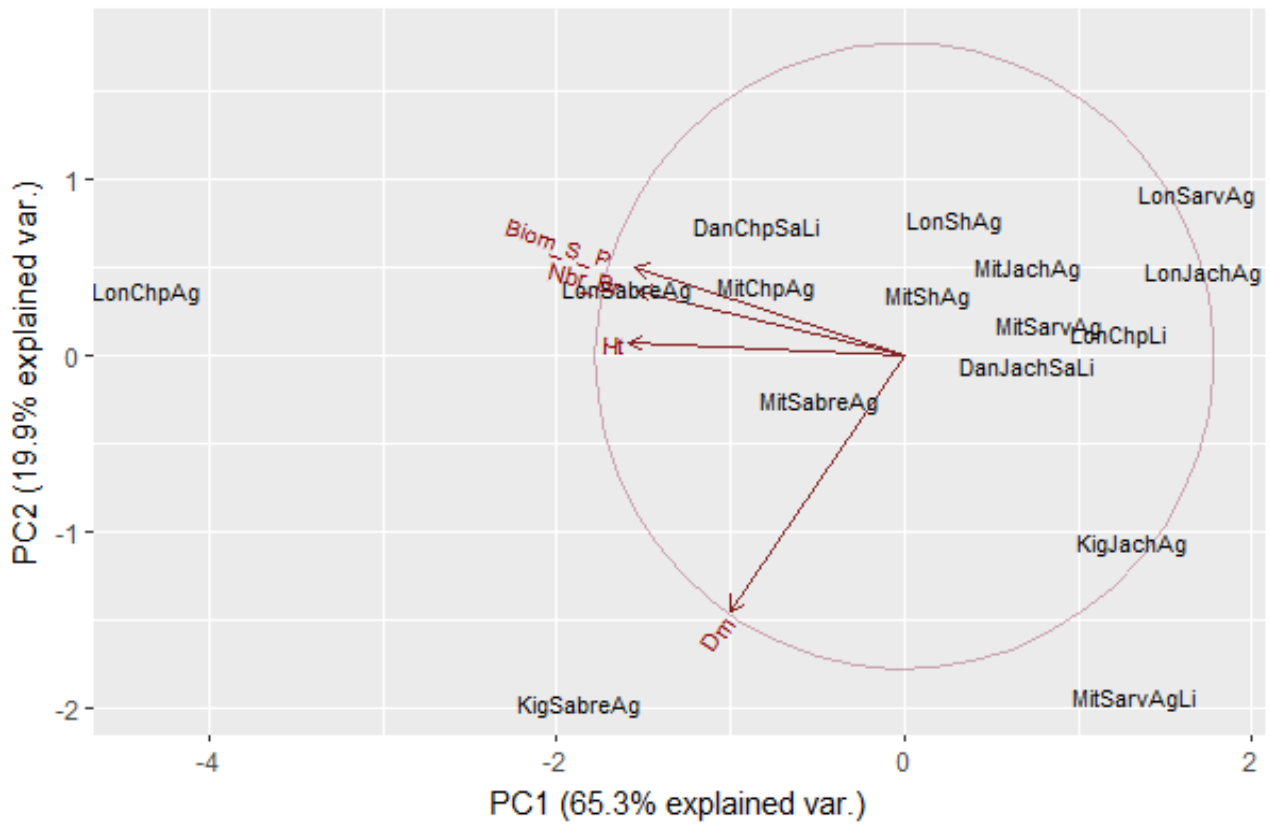

Figure 3 : Relation entre la biomasse des ligneux, les paramètres dendrométriques, la formation végétale et les caractéristiques pédologiques.

\section{DISCUSSION}

Composition floristique des ligneux fourragers : Le cortège floristique du parcours des transhumants de la BMVO et de sa Zl est constitué de 41 espèces ligneuses réparties dans 21 familles et 38 genres avec une forte abondance de la famille des légumineuses ( 11 espèces), des Rubiaceae (5 espèces) et des Euphorbiaceae ( 3 espèces). Cette flore peut être décomposée suivant la fréquence, en espèces plus abondantes Mitragyna inermis qui appartient à la famille des Rubiaceae; celles moyennes abondantes notamment Lonchocarpus sericeus, Pterocarpus santalinoides et Daniellia oliveri appartiennent à la famille des légumineuses; Morinda lucida à la famille des Rubiaceae et Elaeis guineensis à la famille des Arecaceae. Les autres ligneux sont classées dans le groupe des espèces rares. Cette richesse floristique, reste faible comparée à celle enregistrée (54 espèces ligneuses) en zone sahélienne au Burkina Faso (Savadogo et al., 2016), et celle enregistrée dans les parcours communautaires (51 espèces d'arbres réparties en 41 genres et 22 familles) du milieu agropastoral du Sénégal (Sarr et al., 2013). Toutefois, cette richesse est supérieure à celle enregistrée (35 espèces) le long des couloirs de transhumance dans la zone de transition soudano-guinéenne au Bénin (Moussa et al., 2017). Le parcours naturel de la BMVO constitue en période sèche la principale ressource alimentaire du bétail où les bovins locaux et transhumants exploitent une diversité de certains ligneux fourragers pour satisfaire leurs besoins. En effet, le nombre le nombre de ligneux fourragers inventoriés dans la BMVO ( 6 espèces, soit 15\%) est faible en comparaison aux études précédentes, 48 ligneux dans les terres de parcours de la zone soudano-guinéenne du Bénin (Sèwadé, 2016); 27 espèces soit $53 \%$ dans le système agropastoral $d u$ Sénégal (Sarr et al., 2013); 18 ligneux fourragers dénombrés dans la vallée du Rift en Éthiopie (Shenkute et al., 2012) et les 28 espèces recensés dans la région de Wolayta au sud de l'Éthiopie (Geta et al., 2014). Plusieurs facteurs peuvent expliquer ces différences, dont notamment les variations des conditions pédoclimatiques et leurs influences sur la distribution spatiale et géographique des flores (Adomou et al., 2006 ; Sèwadé et al., 2016). Par ailleurs, cette végétation ligneuse de la plaine inondable de la BMVO est sous l'influence de la crue du fleuve Ouémé où l'inondation dure en moyenne 4 à 5 mois et de décrue cycliques. A ces stress hydrique et de sécheresse, il faut ajouter celui créé par le feu de végétation allumé par les éleveurs. Ces perturbations laissent envisager que certains ligneux et certains individus ne pourront pas tolérés ces stress. Ce qui expliqueraient en partie le faible niveau de diversification des ligneux et de leur densité. Assédé et al. (2015) ont rapporté, que le niveau d'asphyxie assez élevé des plaines d'inondation exige des stratégies de survie particulières des espèces. Ce que certaines espèces ou individus n'arrivent probablement à développer. Les travaux précédents ont montré que les formations des plaines d'inondation étaient moins diversifiées que celles des plateaux car les 
écosystèmes de plateaux sont peu sélectifs et offrent des conditions édaphiques plus favorables à l'installation et au développement de plusieurs espèces (Bondé et al., 2013). La rencontre de certains ligneux ayant d'affinité pour l'eau et des conditions édaphiques similaires a été rapporté pour des espèces tels que Mitragyna inermis, Pterocarpus santalinoides (Sambaré et al., 2011; Assédé et al., 2015), Lonchocarpus sericeus (Gbètoho et al. 2017), Daniellia oliveri (Sèwadé et al., 2017 ), Kigelia africana (Bello et al., 2016). Ce qui confirme la présence et le développement de ces ligneux dans cet écosystème de la BMVO.. Par ailleurs, certains ligneux fourragers ayant de faible indice de qualité dans la zone d'étude tels que Mangifera indica (Manguier), Flueggea virosa (Securinega vénéneux), Annona senegalensis (Pomme cannelle du Sénégal), Anacardium occidentale (Pommier cajou), Ceiba pentadra (Kapokier), Anogeissus leicarpus (Bouleau d'Afrique), Azadirachta indica (Neem), Gardenia erubescens, Sarcocephalus latifolius (Pêcher africain), Acacia auriculiformis (Acacia japonais) ont été aussi signalés comme fourragers par d'autres auteurs (Sèwadé et al., 2016). Cette différence s'expliquerait par la disponibilité en période sèche en abondance de bonnes graminées qui sont plus préférées ou par l'effet de l'habitude alimentaire par rapport à ces ligneux.

Mode d'exploitation et production fourragère des ligneux: Un total de $50 \%$ de ligneux fourragers prioritaires appartient à la famille des légumineuses et le reste à celles des Bignoniaceae $(16,67 \%)$, des Rubiaceae $(16,67 \%)$ et des Verbenaceae $(16,67 \%)$. Nos résultats révèlent que Pterocarpus santalinoides et Kigelia africana font l'objet d'émondage pour l'affourragement des ruminants domestiques comme Afzelia africana, Pterocarpus erinaceus, Khaya senegalensis qui sont souvent cités comme principaux ligneux fourragers (Teka et al., 2007 ; Ouédraogo-Kone et al., 2008). K. africana est l'un des rares ligneux nonlégumineuses émondées. En effet, les ligneux fourragers constituent une ressource fourragère d'appoint pendant la période sèche pour les transhumants locaux et transfrontaliers. Ces ligneux sont exploités quand les résidus de récolte sont terminés, le fourrage herbacé devient rare soit à cause de sa lignification, ou de son ravage par le feu de végétation. Pour l'alimentation animale, les feuilles fraîches, sans conservation sont les parties les plus utilisées de ces ligneux. Les modes d'exploitations actuels de Pterocarpus santalinoides et Kigelia africana par les éleveurs, constituent une menace pour ces ligneux, surtout lorsque le feuillage se trouvent hors de porté des animaux comme l'a observé Sarr et al. (2013). Ce mode d'exploitation varie en fonction des espèces. L'exploitation des jeunes repousses de $M$. inermis pourrait indiquer la préférence des jeunes repousses par les ruminants domestiques. En alimentation des gros et petits ruminants, plusieurs travaux ont montré l'importance sur les terres de parcours de ces ligneux fourragers prioritaires tels que Daniellia oliveri (Sèwadé et al., 2017); K. Africana (Ogunbosoye and Babayemi, 2010 ; Ky-Dembele et al., 2016); P. santanoloides (Ky-Dembele et al, 2016; Arigbede et al., 2006;) ; M. inermis (Sarr et al., 2013) ; Lonchocarpus sericeus (Ezeagu et al., 2013) et Vitex doniana (Oumorou et al., 2010 ; Sèwadé et al. 2018). Concernant les caractéristiques dendrométriques, le diamètre moyen du tronc de $D$. oliveri est inférieur à celui enregistré $(31 \mathrm{~cm})$ dans la zone de transition Soudano Guineo-Congolaise du Bénin (Sèwadé et al., 2017) . Les densités enregistrées dans le cadre de cette étude sont supérieures à ceux enregistrés pour $D$. oliveri, Pterocarpus erinaceus et Afzelia africana par les mêmes auteurs. Par ailleurs, les densités de Afzelia africana, Khaya senegalensis et Pterocarpus erinaceus dans le système agropastoral au Sud du Burkina Faso sont plus élevées (Ouédraogo-Koné et al., 2008) que celles enregistrées au cours de cette étude pour des ligneux fourragers prioritaires tels que $D$. oliveri, $K$. africana et Lonchocarpus sericeus. De même, la biomasse foliaire est plus importante dans le système agropastoral au Sud du Burkina Faso quelque soit le type de ligneux. Cette faible densité et production fourragère enregistrées dans la zone d'étude, seraient un témoignage d'un milieu fortement anthropisé dans un contexte de variabilité climatique qu'a connu et connait encore le pays, caractérisé par une longue durée de sécheresse ou un niveau d'eau trop élevé ou trop bas dans la plaine inondable. Ces facteurs anthropiques se traduisent par le surpâturage, l'utilisation répétée de feu de végétation, les pratiques agricoles non adaptées et les coupes des ligneux pour divers usages domestiques. L'utilisation des feuilles de Pterocarpus santalinoides qui est utilisé dans la pharmacopée et pour ses propriétés insecticides (Adeleke et al., 2009; Anowi et al., 2012) et l'utilisation de $M$. inermis utilisées en pharmacopée africaine chez les petits éleveurs dans la lutte contre les parasitoses gastro-intestinales des ruminants (Djoueché et al., 2011) seraient des témoignages d'un usage à but multiple de certains ligneux. L'ingestion des jeunes feuilles de $M$. inermis par les bovins transhumants serait alors dans le but d'un alicament (fourrage et déparasitant). Au Ghana comme dans de nombreuses régions de l'Afrique subsaharienne, la feuille de Kigelia africana est utilisée pour préparer une soupe de noix de palme consommée principalement par les femmes allaitantes (Glew \& Amoako-Atta, 2010). Ces auteurs ont rapporté que les feuilles séchées contiennent d'acides gras (1,62\%), dont l'acide a-linolénique (44\%) 
et l'acide linolénique (20\%). Les protéines représentaient $12,6 \%$ du poids sec et, son profil global en acides aminés essentiels (à l'exception de la lysine) se comparait favorablement à un standard protéique de I'Organisation Mondiale de la Santé pour les écoliers (Glew \& Amoako-Atta, 2010). Ces données indiquent que la feuille de Kigelia africana pourrait aussi stimuler

\section{CONCLUSION}

La présente étude a permis d'avoir une connaissance de l'état des ligneux fourragers de la zone d'accueille des transhumants et le comportement alimentaire des bovins dans l'écosystème de la BMVO. La méthode utilisée a permis d'évaluer la productivité des ligneux des parcours pour une meilleure gestion des ressources utilisées par le bétail. Cette étude révèle l'intérêt pastoral de cet écosystème où les ligneux subissent de manière diversifiée une forte pression de prélèvement. Les transhumants sur les parcours, reconnaissent la valeur des ligneux à travers

\section{RÉFÉRENCES BIBLIOGRAPHIQUES}

Atchade GAA, Vissin EW, Gbenou P, Chabi BIH, Lavinon SRF, 2017. Cultures de contre-saison dans la commune d'Adjohoun au Bénin : Entre Atouts Et Contraintes Revue Ivoirienne de Géographie des Savanes, 2, 172-184.

Adamm KS, Boko M. Le Bénin, Paris: Sodimas-edicef, 96pp.

Adeleke MA, Popoola SA, Agbaje WB, Adeoye B, Adeoye MD, Jimoh WA, 2009. Larvicidal efficacy of seed oils of Pterocarpus santalinoides and Tropical Manihot species against Aedes aegypti and effects on aquatic fauna. Tanzania Journal of Health Research 11 (4), 250-252.

Adomou AC, Sinsin B, Van der Maesen LJG, 2006. Phytosociological and chorological approaches to phytogeography: a study at meso-scale in Benin. Syst. Geogr. Plants 76, 155-178.

Adomou AC, 2005. Vegetation patterns and environmental gradients in Benin. Implications for biogeography and conservation. $\mathrm{PhD}$ Thesis. Wageningen University, Wageningen : 150p.

Agence pour la Sécurité de la Navigation Aérienne en Afrique et au Madagascar (ASECNA), 2016. Données climatiques de température et de pluviométrie dans les stations de Bohicon de1985 à 2012.

Alimi RM, Biaou C, Egboou PB, 2015. Analyse des dynamiques sociales liées a une gestion apaisée de la transhumance dans la Basse et Moyenne Vallée de l'Ouémé et sa zone effectivement la production laitière chez les vaches allaitantes. La capacité de charge des ligneux est faible probablement à cause de leur surexploitation. Toutefois, leur importance en alimentation est élevée car ils apporteraient les nutriments essentiels nécessaires pour valoriser les fourrages de mauvaises qualités de la saison sèche.

l'appétibilité des bovins. Les modes d'exploitation des ligneux fourragers les plus recherchés constitue une menace pour leur conservation et leur régénération naturelle. Par conséquent, ces essences forestières fourragers tels que $D$. oliveri, $K$. africana, $L$. sericeus, $M$. inermis, $P$. santalinoides, $V$. doniana sont prioritaires pour un aménagement. La mise au point de la technique efficace de régénération et le changement de mode d'exploitation sont indispensables pour assurer une durabilité des services sociaux et pastoraux.

d'influence en république du Bénin. Rapport d'étude, SNV-Bénin, 71p.

Anowi CF, Umeokoli BO, Onyegbule AF, Okonkwo C, Chibeze, I, 2012. Analgesic, phytochemical and acute toxicity evaluation of the methanol extract of the leaves of Pterocarpus santalinoides- Family Fabacea. International Journal of Pharmaceutical Science Research 3 (7), 2018-2023.

Arigbede OM, Anele UY, Olanite JA, Adekunle IO, Jolaosho OA, Onifade OS, 2006. Seasonal in vitro gas production parameters of three multi purpose tree species in Abeokuta, Nigeria. Livestock Research for Rural Development 18 (10).

Assédé ESP, Azihou FA, Adomou AC, Oumorou M, Sinsin B, 2015. Effet du relief sur la régénération des espèces ligneuses en zone soudanienne du Bénin. Bois et forêts des tropiques 326 (4).

Bondé L, Ouédraogo $\mathrm{O}$, Kagambèga $\mathrm{F}$, Boussim Jl, 2013. Impact des gradients topographique et anthropique sur la diversité des formations ligneuses soudaniennes. Bois et forêts des tropiques 318 (4).

Breman H, Ridder N, DE Ketelaars JJMH, Keuken VAN H, 1991. Manuel sur les pâturages des pays sahéliens. Paris, Khartala

Coulibaly D., Moulin C.H., Poccard-Chappuis R., Morin G., Sidibé S.I., Corniaux C., 2007. Évolution des stratégies d'alimentation des élevages bovins dans le bassin d'approvisionnement en 
lait de la ville de Sikasso au Mali. Revue Élev. Méd. vét. Pays trop. 60 (1-4) : 103-111.

Djoueché CM, Azebaze AB, Dongmol AB, 2011. Investigation of Plants Used for the ethnoveterinary control of gastrointestinal parasites in Bénoué Region, Cameroon. Tropicultura 29 (4), 205- 211.

Ezeagu IE, Akinsoyinu AO, Tarawali G, 2013. Nutritional factors in some fodder legume trees and shrubs Nigerian. Journal of Animal Science 15, 191-198.

Food and Agriculture Organization of the United Nations (FAO), 2012. La transhumance transfrontalière en Afrique de l'Ouest: Proposition de plan d'action, Organisation des Nations Unies pour l'Alimentation et l'Agriculture, Ouagadougou, $143 \mathrm{p}$.

Gbètoho AJ, Aoudji AKN, Roxburgh L, Ganglo JC, 2017. Évaluation de l'aptitude d'essences pionnières pour la restauration de forêts secondaires au bénin dans un contexte de changement climatique. Bois et Forêts des Tropiques 332 (2).

Geta T, Nigatu L, Animut G, 2014. Évaluation of potential yield and chemical composition of selected indigenous multi-purpose fodder trees in three districts of Wolayta Zone, Southern Ethiopia. World Appl. Sci. J. 31 (3), 399-405.

Ginane C, Dumont B, Baumont R, Prache S, Fleurance G, Farruggia A, 2008. Comprendre le comportement alimentaire des herbivores au pâturage: intérêts pour l'élevage et l'environnement. Rencontre Recherche Ruminants 15, 315-322.

Glew RS, Amoako-Atta B, 2010. Indigenous Plant Food Used by Lactating Mothers in West Africa: The Nutrient Composition of the Leaves of Kigelia Africana in Ghana. Ecology of Food and Nutrition 49 (1), 72-83.

Kiema A, Tontibomma GB, Zampaligré N, 2014. Transhumance et gestion des ressources naturelles au Sahel : contraintes et perspectives face aux mutations des systèmes de productions pastorales. VertigO - la revue électronique en sciences de l'environnement [En ligne], 14 (3) consulté le 26 juin 2017. http://vertigo.revues.org/15404.

Ky-Dembele C, Bayala J, Kalinganire A, Traoré FT, Koné B, Olivier A, 2016: Vegetative propagation of twelve fodder tree species indigenous to the Sahel, West Africa, Southern Forests. Journal of Forest Science 78 (3). http://www.tandfonline.com/eprint//m9gSrEUR gfmgkzYHejS/full

DOI: $10.2989 / 20702620.2016 .1157980$
Lalèyè $P$, Chikou A, Philippart JC, Teugels GG, Vandewalle P, 2004. Étude de la diversité ichtyologique du bassin du fleuve Ouémé au Bénin (Afrique de l'Ouest). Cybium. 28(4): 329-339.

Le S, Josse J, Husson F, 2008. FactoMineR: An Package for Multivariate Analysis. Journal of Statistical Software. 25 (1), 1-18.

Loiseau P, 1988. Signification et limite de l'indice de valeur pastorale pour le diagnostic, de la valeur agricole des pâturages en moyenne montagne humide. XVI Colloques phytosociologiques. 411-428.

Mebirouk-Boudechiche L, Cherif M, Boudechiche L, Sammar F, 2014. Teneurs en composés primaires et secondaires des feuilles d'arbustes fourragers de la région humide d'Algérie L. Revue Méd. Vét. 165, 11-12.

Mendiburu F, 2015. Agricolae: Statistical Procedures for Agricultural Research $\mathrm{R}$ package version 1.2.3 available online at http//CRAN Rproject.org/package-agricolae.

Moussa L, Ismaillal Y, Imorou T, 2017. Diversité floristique et usages des Ligneux le long des couloirs de transhumance dans la commune de Savè au Centre Bénin. European Scientific Journal 13 (2).

Njidda AA. and lkhimioya I, 2010. Correlation Between Chemical Composition and In Vitro Dry Matter Digestibility of Leaves of Semi-Arid Browses of North-easten Nigeria. American-Eurasian J; Agric and Environ. Sci. 9 (2), 169-175.

Ogunbosoy DO. and Babayemi OJ, 2010. Potential values of some non-leguminous browse plants as dry season feed for ruminants. Nigeria African Journal of Biotechnology 9 (18), 2720 2726.

Ouédraogo-Kone S, Kaboré-Zoungrana CY, Ledin I, 2008. Important characteristics of some browse species in an agrosilvopastoral system in West Africa. Agroforest Syst. 74, 213-221.

Oumorou M, Sinadouwirou T, Kiki M, Glele Kakaï R, Mensah GA, Sinsin B, 2010. Disturbance and population structure of Vitex doniana Sw. in northern Benin, West Africa. Int. J. Biol. Chem. Sci. 4 (3), 624-632.

Paradis G. 1983. A phytogeographic survey of Southern Benin. Bothalia 14, 3 \& 4: 579-585.

Pamo ET, Boukila B,. Fonteh FA, Tendonkeng F, Kana JR, Nanda AS, 2007. Nutritive value of some grasses and leguminous tree leaves of the Central region of Africa. Animal Feed Science and Technology. 135, 273-282.

Pelissier $P$, 1963. Les pays du bas-Ouémé : une région témoin du Dahomey méridional. FLASH, 
Dakar, Sénégal. Travaux du département de Géographie. 10, 1-39.

Petit S. and Mallet B, 2001. L'émondage d'arbres fourragers : détail d'une pratique pastorale. Bois et forêts des tropique 270 (4).

R Development Core Team, 2016. R: A Language and Environment for Statistical Computing. R Foundation for Statistical Computing, Vienna.

Sambaré O, Bognounou $F$, Wittig R, Thiombiano A, 2011. Woody species composition, diversity and structure of riparian forests of four watercourses types in Burkina Faso. Journal of Forestry Research 22 (2), 145-158.

Sarr O, Diatta S, Gueye M, Ndiaye PM, Guisse A, Akpo LE, 2013. Importance des ligneux fourragers dans un système agropastoral au Sénégal (Afrique de l'ouest). Revue Méd. Vét. 164 (1) 2-8.

Sarr O, Ngom D, Ngaryo FD, Diatta S, Akpo LE, 2014. État de la végétation ligneuse dans trois unités d'utilisation des terres d'une zone agropastorale au Sénégal (région de Kaffrine). Int. J. Biol. Chem. Sci. 8 (6), 2669-2684.

Savadogo OM, Ouattara K, Pare S, Ouedraogo I, Kaboré SS, Barron J, Zombre NP, 2016 Structure, composition spécifique et diversité des ligneux dans deux zones contrastées en zone Sahélienne du Burkina Faso. Vertigo 16 (1).

Sèwadé $C$, Azihou $A F$, Fandohan $A B$, Glèlè Kakaï RL, Mensah GA, Houinato MRB, 2017. Leaf biomass modeling, carrying capacity and species-specific performance in aerial fodder production of three priority browse species Afzelia africana, Pterocarpus erinaceus and Daniellia oliveri in Benin. Livestock Research for Rural Development 29, 192 http://www.Irrd.org//rrd29/10/sewa29192. html

Sèwadé C, Azihou AF, Fandohan AB, Houéhanou DT, Houinato M, 2016. Diversité, priorité pastorale et de conservation des ligneux fourragers des terres de parcours en zone soudanoguinéenne du Bénin. Biotechnologie Agronomie Société et Environnement. 20 (2), 113-129. http://popups.ulg.ac.be/1780-4507/ index. php?id=12823.

Sèwadé C, Lokonon BE., Azihou AF, Akouèhou GS, Mensah GA. Glèlè Kakaï RL, Houinato M. R. B., 2018. Use diversity and farmer's preference of 48 local multipurpose fodder trees: a comparative analysis of three sociolinguistic groups of Benin. Annales des sciences agronomiques. 22 (1), 33-52.
Shenkute B, Hassen A, Assafa T, Amen N, Ebro A, 2012. Identification and nutritive value of potential fodder trees and shrubs in the mid rift valley of Ethiopia. Journal of Animal \& Plant Sciences 22 (4), 1126-1132

Sidi Imorou $\mathrm{H}$, Babatounde $\mathrm{S}$, Sidi Imorou $\mathrm{F}$, Mensah GA, 2016. Ligneux fourragers des parcours naturels communautaires du Nord-Bénin: prédiction de la valeur nutritive au moyen de plusieurs approches analytiques. Journal of Animal \&Plant Sciences 29 (1), 4537-4552.

Sinsin B, 1993. Phytosociologie, écologie, valeur pastorale, productivité et capacité de charge des pâturages naturels du périmètre NikkiKalalé au Nord Bénin. Thèse de doctorat, Univ. Libre de Bruxelles, Belgique, $390 \mathrm{p}$.

Teka O, Vogt J, Sinsin B, 2007. Impacts de l'élevage sur les ligneux fourragers et contribution à la gestion intégrée de Khaya senegalensis et Afzelia africana, deux espèces menacées d'extinction dans la région des Monts-Kouffé au Bénin. Bulletin Recherche Agronomique Bénin 55, 25-35.

Zampaligré N, Dossa LH, Schlecht E, 2013. Contribution of browse to ruminant nutrition across three agro-ecological zones of Burkina Faso. Journal of Arid Environments 95, 55-64. 